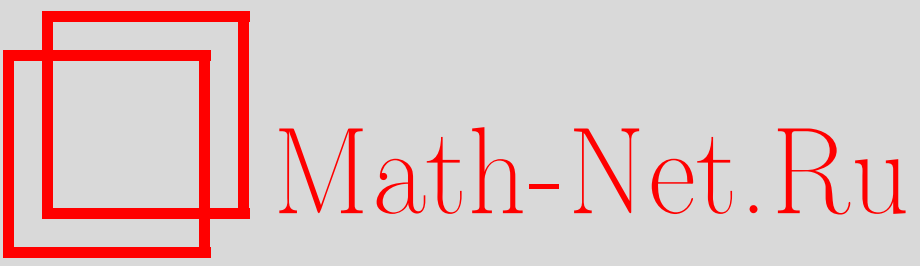

А. А. Пожарский, Кристалл с сингулярным потенциалом в однородном электрическом поле, ТМФ, 2000, том 123, номер 1, 132-149

DOI: https://doi.org/10.4213/tmf592

Использование Общероссийского математического портала Math-Net.Ru подразумевает, что вы прочитали и согласны с пользовательским соглашением

http://www . mathnet.ru/rus/agreement

Параметры загрузки:

IP: 35.174 .16 .151

26 апреля 2023 г., 02:50:49 
ТЕОРЕТИЧЕСКАЯ

И МАТЕМАТИЧЕСКАЯ

ФИЗИКА

Том 123, № 1

апрель, 2000

(C) 2000 r.

А. А. Пожарский*

\section{КРИСТАЛЛ С СИНГУЛЯРНЫМ ПОТЕНЦИАЛОМ В ОДНОРОДНОМ ЭЛЕКТРИЧЕСКОМ ПОЛЕ}

Изучаются асимптотические поведения решений одномерного уравнения Шредингера $-\psi^{\prime \prime}+q(x) \psi-F x \psi=E \psi$ при больших значениях аргумента. Потенциал $q$ предполагается периодической функцией, абсолютно интегрируемой на периоде. Показано, что спектральная проблема для исходного уравнения Шредингера может быть сведена к спектральной проблеме для дискретной системы. В случае гладкого потенциала $q$ матрица перехода быстро стремится к единичной. Если потенциал $q$ не является гладким, то матрица перехода медленно стремится к единичной, а дискретная система проявляет случайные свойства. Это объясняет, почему природа спектра исходного уравнения до сих пор практически не изучена.

\section{1. ВВЕДЕНИЕ}

Рассмотрим одномерное движение квантового электрона в периодическом кристалле, помешенном в однородное электрическое поле. Это движение описывается уравнением

$$
-\psi^{\prime \prime}+q(x) \psi+v(x) \psi=E \psi
$$

Здесь $x \in \mathbb{R}, \psi=\psi(x, E) \in \mathbb{C}, q(x)$ - вешественньй периодический потенциал:

$$
q(x+a)=q(x)
$$

$v(x)$ - потенциал, описываюший однородное электрическое поле:

$$
v(x)=-F x
$$

Если потенциал $q$ является гладким, то природа спектра уравнения (1.1) хорошо изучена [1-6]. Например, известно, что если $q$ имеет две непрерывные производные, то уравнение (1.1) имеет однократный абсолютно непрерывный спектр, заполняющий всю вешественную ось. Однако если потенциал $q$ не является гладким, то вопрос о природе

\footnotetext{
* Санкт-Петербургский государственный университет, Санкт-Петербург, Россия
} 
спектра до сих пор остается открытым, имеются лишш некоторые частные результаты. Так, доказано, что для потенциала

$$
q(x)=V \sum_{-\infty}^{+\infty} \delta^{\prime}(x-n)
$$

спектр уравнения (1.1) не содержит абсолютно непрерывной компоненты [7].

Если потенциал

$$
q(x)=V \sum_{-\infty}^{+\infty} \delta(x-n)
$$

то спектр уравнения (1.1) зависит от числа $\pi^{2} / F$ и до конца не изучен. Исследованию этого случая посвящена работа [8].

Случайный сингулярный потенциал изучается в работе [9], в которой рассмотрен случайный потенциал $q$, заданный в виде

$$
q(x)=\sum_{-\infty}^{+\infty} V_{n} \delta(x-n)
$$

где коэффициены $V_{n}$ случайно идентично распределены и их среднее значение равно нулю. Авторы смогли описать природу спектра этой модели и найти ее зависимость от параметров модели.

Данная работа посвящена исследованию случая, когда потенциал $q$ является интегрируемой функцией на периоде

$$
q(x) \in L_{1}[0, a]
$$

Для упрощения формул будем предполагать, что $F>0$ и

$$
\int_{0}^{a} q(t) d t=0
$$

последнего всегда можно добиться сдвигом спектрального параметра $E$.

В этой работе изучаются асимптотические поведения решений уравнения (1.1) при $x \rightarrow+\infty$. Показано, что на интервалах $I_{l} \subset\left(\widetilde{n}_{l-1} a, \widetilde{n}_{l} a\right)$, где

$$
\widetilde{n}_{l}=\frac{(\pi l)^{2}}{F a^{3}}-\frac{E}{F a}
$$

произвольное решение $\psi$ может быть описано асимптотической формулой

$$
\psi(x) \sim \frac{s_{l}}{\sqrt[4]{F x}} \exp \left\{i \frac{2}{3 F}(E+F x)^{\frac{3}{2}}\right\}+\frac{t_{l}}{\sqrt[4]{F x}} \exp \left\{-i \frac{2}{3 F}(E+F x)^{\frac{3}{2}}\right\}
$$


при $l \rightarrow \infty$. Здесь $s_{l}$ и $t_{l}-$ константы. Длина интервалов $I_{l}$ растет как $2 \pi^{2} l /\left(F a^{2}\right)$ при $l \rightarrow \infty$. Формула (1.2) неверна в относительно малых окрестностях точек $\widetilde{n}_{l}$, более точно - в окрестностях порядка $l^{1 / 2}$. Несмотря на это, справедлива асимптотическая формула

$$
\int_{\tilde{n}_{l-1} a}^{\tilde{n}_{l} a}|\psi(x)|^{2} d x \sim \frac{2 \pi}{F a}\left\|\mathbf{s}_{l}\right\|^{2}, \quad \mathbf{s}_{l}=\left(\begin{array}{c}
s_{l} \\
t_{l}
\end{array}\right) .
$$

В работе также определено асимптотическое поведение решений при $l \rightarrow \infty$ в окрестностях точек поворота $\widetilde{n}_{l}$. Благодаря этому устанавливается связь между асимптотическими формулами (1.2) на смежных интервалах $I_{l}$ и $I_{l+1}$. Эта связь дается преобразованием

$$
\mathbf{s}_{l+1}=W_{l} \mathbf{s}_{l}, \quad l \in \mathbb{N},
$$

где

$$
\begin{gathered}
W_{l}=e^{-i \Gamma_{l} \sigma_{3}} S_{l} e^{i \Gamma_{l} \sigma_{3}}, \quad \Gamma_{l}=-\frac{1}{3 F}\left(\frac{\pi l}{a}\right)^{3}+\pi l \frac{E}{F a} \\
S_{l}=\left(\begin{array}{cc}
1+q(l) l^{-1} & r(l) l^{-\frac{1}{2}}\left(1+O\left(l^{-\frac{1}{2}}\right)\right) \\
\bar{r}(l) l^{-\frac{1}{2}}\left(1+O\left(l^{-\frac{1}{2}}\right)\right) & 1+\bar{q}(l) l^{-1}
\end{array}\right) .
\end{gathered}
$$

Здесь $q$ и $r$ - комплексные функции:

$$
\begin{aligned}
& r(l)=-i e^{-i \frac{\pi}{4}} \frac{1}{(2 F a)^{\frac{1}{2}}} \int_{0}^{a} q(t) e^{-i \frac{2 \pi l}{a} t} d t \\
& q(l)=\frac{1}{2 \pi F a}\left|\int_{0}^{a} q(t) e^{-i \frac{2 \pi l}{a} t} d t\right|^{2} \int_{-\infty}^{+\infty} e^{-i t^{2}} d t \int_{-\infty}^{t} e^{i s^{2}} d s
\end{aligned}
$$

удовлетворяющие условию

$$
q(l)+\bar{q}(l)=|r(l)|^{2}
$$

Не следует путать $q(l)$ в этих формулах с потенциалом $q(x)$ в исходном уравнении.

Здесь и далее для упрошения записи введено предположение о том, что коэффициент $r(l)$ убывает не быстрее чем $O\left(l^{-1}\right)$. Заметим, что в случае более быстрого убывания $r(l)$ матрица $S_{l}$ имеет вид

$$
S_{l}=\left(\begin{array}{cc}
1+O\left(l^{-3}\right) & O\left(l^{-\frac{3}{2}}\right) \\
O\left(l^{-\frac{3}{2}}\right) & 1+O\left(l^{-3}\right)
\end{array}\right)
$$

Легко видеть, что $W_{l}$ является $F a$-периодической функцией от $E$. Это соответствует $F a$-периодичности спектра исходного уравнения.

Спектральное значение точек поворота $\widetilde{n}_{l}$ вполне ясно. Далекие лакуны в спектре чисто периодического уравнения

$$
-\psi^{\prime \prime}+q(x) \psi=\mathcal{E} \psi
$$


располагаются около точек $\mathcal{E}=(\pi l / a)^{2}$. Поэтому точки поворота $\widetilde{n}_{l}$, удовлетворяюшие уравнению $\left(E+F a \widetilde{n}_{l}\right)^{1 / 2} a=\pi l$, могут быть ассоциированы с лакунами [1]. Таким образом, можно сказать, что матрица $W_{l}$ описывает переход через окрестность лакуны с номером $l$.

Хотя эта работа мотивирована спектральными вопросами, она не содержит никаких законченных спектральных результатов. Несмотря на это, хотелось бы сделать несколько замечаний о возможной связи полученных здесь результатов с вопросами спектральной теории.

Известно, что спектральные свойства уравнения (1.1) могут быть [10] характеризованы асимптотическим поведением интеграла

$$
\int_{x_{0}}^{x}|\psi(x)|^{2} d x
$$

Это значит, что спектральные свойства уравнения (1.1) можно понять через спектральные свойства системы (1.4) [11].

В целом необходимо подчеркнуть, что обсуждаемая здесь связь спектральных свойств задач (1.1) и (1.4) сама по себе еше не содержит ответа на вопрос о природе спектра для какой-либо из этих задач. Хотя система (1.4) выглядит гораздо более контролируемым объектом в сравнении с (1.1), изучение асимптотических свойств решений системы (1.4) при $l \rightarrow \infty$ является, бесспорно, трудной задачей гармонического анализа. Например, в старшем порядке теории возмушений асимптотика решений системы (1.4) описывается поведением сложных тригонометрических сумм вида

$$
\sum_{l=1}^{L} \frac{r(l)}{\sqrt{l}} e^{-2 i \Gamma_{l}}
$$

Впрочем, сфформулируем следующую лемму.

ЛЕмма 1. Пусть потенииал q удовлетворяет оценке

$$
\left|\int_{0}^{a} q(t) e^{i \frac{2 \pi l}{a} t} d t\right|<\mathrm{const} \cdot l^{-\frac{1}{2}-\delta}, \quad l=1,2, \ldots,
$$

при некотором $\delta>0$. Тогда уравнение (1.1) имеет однократный абсолютно непрерывньй спектр, заполняющий всю вещественную ось.

Доказательство леммы опирается на теорему из работы [10]. Мы планируем опубликовать ее детальное доказательство отдельно, т.к. оно не помешается в допустимые рамки данной работы.

Все выкладки в этой работе вполне просты, но не являются короткими, поэтому некоторые из них опушены. В частности, акцентируется внимание на построении формального асимптотического решения уравнения (1.1), но не обсуждается сушествование точного решения этого уравнения, чье асимптотическое поведение описывается построенным формальным решением. Доказательство этого факта также вполне элементарно, но не коротко. 
Во втором разделе статьи задача о поиске решения уравнения (1.1) сводится к дискретной модели, описывающей переход через точки na. Затем строятся асимптотические решения этой задачи вне малых окрестностей точек поворота, а именно на интервалах $I_{l}$. В третьем разделе строятся асимптотические решения этой же задачи в окрестностях точек поворота. Четвертый раздел посвящен нахождению преобразования, с помошью которого связаны асимптотические решения дискретной задачи на смежных интервалах $I_{l}$ и $I_{l+1}$. В последнем разделе найдена аналитическая структура асимптотического решения уравнения (1.1) при $x \rightarrow+\infty$ на интервалах $I_{l}$ и определена связь коэффициентов, от которых зависит структура асимптотического решения, на смежных интервалах $I_{l}$ и $I_{l+1}$.

\section{2. АДИАБАТИЧЕСКИЙ ПОДХОД}

Этот раздел посвящен построению асимптотических решений уравнения (1.1) вне малых окрестностей точек поворота. Возможность асимптотического описания решений основана на адиабатических соображениях. Мы следуем здесь работе [8].

Полагая $p(x)=q(x)-F x$, уравнение (1.1) можно переписать в виде

$$
\psi^{\prime \prime}+[E-p(x)] \psi=0 .
$$

Пусть $\theta(x, E)$ и $\varphi(x, E)$ - такие решения уравнения $(2.1)$, что

$$
\left\{\begin{array} { l } 
{ \theta ( 0 , E ) = 1 , } \\
{ \theta ^ { \prime } ( 0 , E ) = i E ^ { \frac { 1 } { 2 } } , }
\end{array} \quad \left\{\begin{array}{l}
\varphi(0, E)=i E^{-\frac{1}{2}} \\
\varphi^{\prime}(0, E)=1 .
\end{array}\right.\right.
$$

Для краткости обозначим функции $\theta(a, E+F a n), \theta^{\prime}(a, E+F a n), \quad \varphi(a, E+F a n)$, $\varphi^{\prime}(a, E+F a n)$ соответственно через $\theta_{n}, \theta_{n}^{\prime}, \varphi_{n}, \varphi_{n}^{\prime}$. При этом

$$
\theta_{n} \varphi_{n}^{\prime}-\theta_{n}^{\prime} \varphi_{n}=W(\theta, \varphi)=2
$$

На интервале $[n a,(n+1) a]$ произвольное решение уравнения $(2.1)$ может быть представлено в виде

$$
\psi(x)=\alpha_{n} \theta(x-n a, E+F a n)+\beta_{n} \varphi(x-n a, E+\text { Fan }) .
$$

Введем вектор

$$
a(n)=\left(\begin{array}{c}
\alpha_{n} \\
\beta_{n}
\end{array}\right)
$$

Легко видеть, что

$$
a(n+1)=M(n) a(n),
$$

где

$$
M(n)=\left(\begin{array}{cc}
\frac{1}{2} \theta_{n}+\frac{1}{2 i k_{n+1}} \theta_{n}^{\prime} & \frac{1}{2} \varphi_{n}+\frac{1}{2 i k_{n+1}} \varphi_{n}^{\prime} \\
-\frac{i k_{n+1}}{2} \theta_{n}+\frac{1}{2} \theta_{n}^{\prime} & -\frac{i k_{n+1}}{2} \varphi_{n}+\frac{1}{2} \varphi_{n}^{\prime}
\end{array}\right), \quad k_{n}=\sqrt{E+F a n} .
$$

Непосредственным вычислением проверяется, что $\operatorname{det} M(n)=1$. 
Положим

$$
\hat{a}(n)=e^{\Phi_{n} \sigma_{3}} a(n), \quad \Phi_{n}=\frac{1}{2} \ln k_{n}, \quad \sigma_{3}=\left(\begin{array}{cc}
1 & 0 \\
0 & -1
\end{array}\right) .
$$

Тогда

$$
\hat{a}(n+1)=T(n) \hat{a}(n),
$$

где

$$
\begin{gathered}
T(n)=e^{i \Delta(n) \sigma_{3}} t(n) \\
t(n)=\left(\begin{array}{cc}
1+\zeta_{n}+O\left(k_{n}^{-3}\right) & \frac{b_{n}}{2 k_{n}}+O\left(k_{n}^{-2}\right) \\
\frac{a_{n}}{2 k_{n}}+O\left(k_{n}^{-2}\right) & 1+\xi_{n}+O\left(k_{n}^{-3}\right)
\end{array}\right), \quad \Delta(n)=k_{n} a+\frac{F a^{2}}{4 k_{n}} \\
\zeta_{n}=\bar{\xi}_{n}=\frac{1}{4 k_{n}^{2}} \int_{0}^{a} q(t) d t \int_{0}^{t} q(y) e^{2 i k_{n}(y-t)} d y \\
a_{n}=\bar{b}_{n}=\int_{0}^{a} q(t) e^{2 i k_{n} t} d t
\end{gathered}
$$

Нетрудно видеть, что выполнено соотношение

$$
\zeta_{n}+\xi_{n}=\frac{a_{n} b_{n}}{4 k_{n}^{2}}
$$

Для больших $n$ уравнение (2.2) может быть решено адиабатически. Это значит, что асимптотически решение может быть представлено в виде

$$
\hat{a}(n)=e^{i \Theta(n)} b(n),
$$

где $b$ и $\Theta$ - гладкие функции параметра $n$ и, кроме того, $b$ - медленно меняющаяся функция. Мы можем найти такие адиабатические решения везде, за исключением относительно малых окрестностей точек поворота $\widetilde{n}_{l}$.

Построим адиабатическое решение. Подставим представление (2.3) в уравнение (2.2):

$$
e^{i \chi(n)} b(n+1)=T(n) b(n),
$$

где

$$
\chi(n)=\Theta(n+1)-\Theta(n) .
$$

Для того чтобы учесть медленную изменяемость $b(n)$, заменим уравнение (2.4) уравнением

$$
e^{i \chi(n)} b(n+\varepsilon)=T(n) b(n),
$$

содержашим вспомогательный параметр $\varepsilon$. Функцию $b(n)$ будем искать в виде формального ряда

$$
b(n)=\sum_{p \geqslant 0} \varepsilon^{p} b_{p}(n)
$$


Соответственно функция $b(n+\varepsilon)$ должна быть разложена в формальный ряд Тейлора. Затем, приравнивая коэффициенты при одинаковых степенях $\varepsilon$, получим уравнения на коэффициенты $b_{p}(n)$. Решив эти уравнения и положив $\varepsilon=1$, найдем ряд для $b(n)$, который является асимптотическим при $n \rightarrow \infty$. Приравнивая коэффициенты при $\varepsilon^{s}$, получим следующее уравнение:

$$
\left(T(n)-e^{i \chi(n)}\right) b_{s}(n)=e^{i \chi(n)} \sum_{p=0}^{s-1} \frac{b_{p}^{(s-p)}(n)}{(s-p) !} .
$$

При $s=0$ данное уравнение имеет вид

$$
e^{i \chi(n)} b_{0}(n)=T(n) b_{0}(n)
$$

Это значит, что $b_{0}(n)$ - собственный вектор и ехр $i \chi(n)$ - соответствуюшее собственное значение матрицы $T(n)$. Последнее уравнение имеет нетривиальное решение тогда и только тогда, когда

$$
\operatorname{det}\left(T-e^{i \chi}\right)=0
$$

что эквивалентно условию

$$
\cos \chi=\frac{1}{2} \operatorname{tr} T=\cos \Delta(n)+\frac{\zeta_{n}+\xi_{n}}{2} \cos \Delta(n)-\frac{\zeta_{n}-\xi_{n}}{2 i} \sin \Delta(n)+O\left(\frac{1}{k_{n}^{3}}\right) .
$$

Вне окрестностей нулей функции $\sin k_{n} a$ легко выделить два решения последнего уравнения:

$$
\begin{aligned}
& \chi^{ \pm}= \pm\left(\Delta(n)-\frac{\zeta_{n}+\xi_{n}}{2} \operatorname{ctg} \Delta(n)+\frac{\zeta_{n}-\xi_{n}}{2 i}+O\left(\frac{1}{k_{n}^{3} \sin \Delta(n)}\right)\right), \\
& \chi^{ \pm}= \pm\left(\Delta(n)-\frac{\zeta_{n}+\xi_{n}}{2} \operatorname{ctg} k_{n} a+\frac{\zeta_{n}-\xi_{n}}{2 i}+O\left(\frac{1}{k_{n}^{3} \sin ^{2} k_{n} a}\right)\right) .
\end{aligned}
$$

Данное представление справедливо вне окрестностей нулей $\sin k_{n} a$ (в точках, где $\sin k_{n} a=0$, оно не сушествует), но мы должны построить асимптотический ряд для $b(n)$. Построим его и покажем, что он является асимптотическим на интервалах

$$
I_{l}=\left(\widetilde{n}_{l-1} a, \widetilde{n}_{l} a\right) \backslash\left(\tilde{i}_{l-1} \cup \tilde{i}_{l}\right),
$$

где окрестности $\tilde{i}_{l}$ задаются неравенствами

$$
\left|n-\widetilde{n}_{l}\right| \leqslant l^{\frac{1}{2}} f^{-1}(l), \quad l^{-\delta} \leqslant f(l)=o(1), \quad 0<\delta \ll 1 .
$$

Проведем соответствуюшие построения для решения $\chi=\chi^{+}$. Для $\chi=\chi^{-}$все можно сделать аналогично.

Удобно ввести собственный вектор $\eta$ сопряженной матрицы:

$$
\begin{aligned}
&\left(T-e^{i \chi}\right)^{*} \eta=0, \\
& \eta=\left(\begin{array}{c}
1 \\
\bar{\gamma}
\end{array}\right), \quad \gamma=\frac{e^{-i \chi}-\bar{t}_{11}}{\bar{t}_{21}} \sim e^{2 i k_{n} a} i \frac{b_{n}}{4 k_{n}} \operatorname{ctg} k_{n} a .
\end{aligned}
$$


При $s=0$ уравнение (2.6) имеет вид

$$
\left(T-e^{i \chi}\right) b_{0}=0
$$

Решая его, найдем

$$
b_{0}=\left(\begin{array}{c}
\alpha_{0} \\
\beta_{0}
\end{array}\right), \quad \beta_{0}=\frac{e^{i \chi}-t_{11}}{t_{12}} \alpha_{0}=\alpha^{+} \alpha_{0}
$$

где

$$
\alpha^{+}=\frac{e^{i \chi^{+}}-t_{11}}{t_{12}}=-i \frac{a_{n}}{4 k_{n}} \operatorname{ctg} k_{n} a+O\left(\frac{1}{k_{n}}\right), \quad \alpha^{+\prime}=i \frac{F a^{2} a_{n}}{k_{n}^{2} \sin ^{2} k_{n} a}+O\left(\frac{1}{k_{n}^{2}}\right) .
$$

При $s=1$ уравнение (2.6) имеет вид

$$
\left(T-e^{i \chi}\right) b_{1}=e^{i \chi} b_{0}^{\prime}
$$

Для его разрешимости необходимо и достаточно, чтобы векторы $b_{0}^{\prime}$ и $\eta$ были ортогональны, т.е.

$$
\left(b_{0}^{\prime}, \eta\right)=\alpha_{0}^{\prime}+\gamma \beta_{0}^{\prime}=0 .
$$

Подставляя сюда $\beta_{0}$ из $(2.11)$ и учитывая $(2.10)$, получим уравнение на $\alpha_{0}$

$$
\alpha_{0}^{\prime}\left(1+\alpha^{+} \gamma\right)+\alpha_{0} \alpha^{+\prime} \gamma=0
$$

Решая его, найдем

$$
\alpha_{0}=\exp \left(-\int_{n_{0}}^{n} \frac{\alpha^{+\prime} \gamma}{1+\alpha^{+} \gamma} d n\right)
$$

где $n_{0}$ и $n_{1}$ - границы интервала $I_{l}$.

Учитывая оценки

$$
\begin{aligned}
\left|\sin ^{-1} k_{n} a\right| & \leqslant C\left(\left(k_{n} a-\pi(l-1)\right)^{-1}+\left(\pi l-k_{n} a\right)^{-1}\right), \\
k_{n}^{-1} & \leqslant C l^{-1}, \quad\left|\sin ^{-1} k_{n} a\right| \leqslant C l^{\frac{1}{2}} f(l)
\end{aligned}
$$

справедливые на интервале $I_{l}$ в силу формул (2.8) и (2.9), оценим интеграл

$$
\int_{n_{0}}^{n} \frac{\alpha^{+\prime} \gamma}{1+\alpha^{+} \gamma} d n=\int_{n_{0}}^{n} \alpha^{+\prime} \gamma d n\left(1+o\left(l^{-\frac{1}{2}}\right)\right)
$$

Легко видеть, что

$$
\begin{gathered}
\left|\int_{n_{0}}^{n} \alpha^{+\prime} \gamma d n\right| \leqslant \int_{n_{0}}^{n_{1}} \frac{D\left|a_{n}\right|^{2}}{k_{n}^{3}\left|\sin k_{n} a\right|^{3}} d n \leqslant \frac{f(l)}{l^{\frac{5}{2}}} \int_{n_{0}}^{n_{1}} \frac{1}{\sin ^{2} k_{n} a} d n, \\
\int_{n_{0}}^{n_{1}} \frac{1}{\sin ^{2} k_{n} a} d n \leqslant C \int_{n_{0}}^{n_{1}}\left(\left(k_{n} a-\pi(l-1)\right)^{-2}+\left(\pi l-k_{n} a\right)^{-2}\right) d n,
\end{gathered}
$$


где $D$ - некоторая постоянная. Учитывая, что

$$
n_{0}=\widetilde{n}_{l-1}+l^{\frac{1}{2}} f^{-1}(l), \quad n_{1}=\widetilde{n}_{l}-l^{\frac{1}{2}} f^{-1}(l),
$$

нетрудно оценить последний интеграл, откуда имеем

$$
\int_{n_{0}}^{n_{1}} \frac{1}{\sin ^{2} k_{n} a} d n=O\left(l^{\frac{3}{2}} f(l)\right), \quad \int_{n_{0}}^{n} \frac{\alpha^{+\prime} \gamma}{1+\alpha^{+} \gamma} d n=O\left(l^{-1} f^{2}(l)\right) .
$$

Теперь с учетом $(2.11),(2.13)$ и (2.16) найдем, что

$$
\alpha_{0}=1+O\left(l^{-1} f^{2}(l)\right), \quad \beta_{0}=O\left(l^{-\frac{1}{2}} f(l)\right) .
$$

Таким образом, найден старший член формального ряда для $b^{+}(n)$. Оценку следуюшим членам формального ряда дает лемма.

Лемма 2. На интервалах $I_{l}$, заданнъх в (2.8) и (2.9), при $l \rightarrow \infty$ справедливь оценки

$$
\begin{array}{ll}
\alpha_{0}^{+}=1+O\left(l^{-1} f^{2}(l)\right), \quad \beta_{0}^{+}=O\left(l^{-\frac{1}{2}} f(l)\right), \\
\alpha_{p}^{+}=O\left(l^{-1} f^{2+2 p}(l)\right), \quad \beta_{p}^{+}=O\left(l^{-\frac{1}{2}} f^{1+2 p}(l)\right), \quad p \geqslant 1 .
\end{array}
$$

Здесь введено следуюшее обозначение:

$$
b^{+}(n)=\sum_{p \geqslant 0} b_{p}^{+}(n), \quad b_{p}^{+}=\left(\begin{array}{c}
\alpha_{p}^{+} \\
\beta_{p}^{+}
\end{array}\right), \quad p \geqslant 1 .
$$

Доказательство этой леммы может быть проведено с помощью метода математической индукции.

Аналогичная лемма справедлива для формального ряда

$$
b^{-}(n)=\sum_{p \geqslant 0} b_{p}^{-}(n), \quad b_{p}^{-}=\left(\begin{array}{c}
\alpha_{p}^{-} \\
\beta_{p}^{-}
\end{array}\right), \quad p \geqslant 1 .
$$

Лемма 3. На интервалах $I_{l}$, заданных в (2.8) и (2.9), при $l \rightarrow \infty$ справедливь оценки

$$
\begin{array}{ll}
\alpha_{0}^{-}=O\left(l^{-\frac{1}{2}} f(l)\right), & \beta_{0}^{-}=1+O\left(l^{-1} f^{2}(l)\right), \\
\alpha_{p}^{-}=O\left(l^{-\frac{1}{2}} f^{1+2 p}(l)\right), & \beta_{p}^{-}=O\left(l^{-1} f^{2+2 p}(l)\right), \quad p \geqslant 1 .
\end{array}
$$

Из (2.5) и (2.7) найдем функции $\Theta^{ \pm}(n)$ :

$$
\Theta^{ \pm}(n)= \pm \Theta(n), \quad \Theta(n)=\frac{2}{3 F} k_{n}^{3}+O\left(l^{-\frac{1}{2}}\right) .
$$

Таким образом, мы построили адиабатические решения уравнения (2.2)

$$
\hat{a}_{l}^{(1)}(n)=e^{i \Theta^{+}(n)} e_{1}, \quad e_{1}=\left(\begin{array}{l}
1 \\
0
\end{array}\right), \quad \hat{a}_{l}^{(2)}(n)=e^{i \Theta^{-}(n)} e_{2}, \quad e_{2}=\left(\begin{array}{l}
0 \\
1
\end{array}\right), \quad n \in I_{l},
$$

при $l \rightarrow \infty$. 
ТеОрема 1. На интервалах $I_{l}$, заданных в (2.8) и (2.9), уравнение (2.2) имеет решения $\hat{a}_{l}^{(1)}(n)$ и $\hat{a}_{l}^{(2)}(n)$, удовлетворяющие следующим условиям:

1) $\hat{a}_{l}^{(1)}(n)=e^{i \Theta^{+}(n)} b^{+}(n), \quad \hat{a}_{l}^{(2)}(n)=e^{i \Theta^{-}(n)} b^{-}(n)$;

2) $\Theta^{ \pm}(n)= \pm \frac{2}{3 F} k_{n}^{3}+O\left(l^{-\frac{1}{2}}\right)$;

3) функиии $b^{+}(n)$ и $b^{-}(n)$ допускают асимптотические разложския

$$
b^{+}(n) \sim \sum_{p \geqslant 0} b_{p}^{+}(n), \quad b^{-}(n) \sim \sum_{p \geqslant 0} b_{p}^{-}(n),
$$

причем функиии $b_{p}^{+}(n)$ и $b_{p}^{-}(n)$ удовлетворяют оценкам (2.17)-(2.20) соответственно.

Доказательство этой теоремы достаточно элементарно [12], и мы его опускаем.

\section{3. ТОЧКИ ПОВОРОТА}

Этот раздел посвяшен построению асимптотических решений уравнения (2.2) в относительно малых окрестностях точек поворота.

Вернемся к уравнению (2.2), переписав его в виде

$$
\hat{a}(n+1)=e^{i \Delta(n) \sigma_{3}} t(n) \hat{a}(n)
$$

Рассмотрим точки поворота $\widetilde{n}_{l}$, которые определены равенствами

$$
k_{\widetilde{n}_{l}} a=\pi l, \quad l \in \mathbb{N} .
$$

Точки $\widetilde{n}_{l}$ не являются целыми, поэтому введем целое число $n_{l}$, ближайшее к $\widetilde{n}_{l}$. Рассмотрим уравнение (3.1) на интервалах $i_{l}$, более широких чем $\tilde{i}_{l}$, а именно в точках $n=n_{l}+m, m \in \mathbb{Z}:$

$$
|m| \leqslant l^{\frac{1}{2}} f^{-2}(l)
$$

где $f(l)$ вводится согласно (2.9).

Основная идея решения уравнения (3.1) состоит в том, чтобы заменить $\Delta(n)$ и $t(n)$ на первые члены рядов Тейлора в окрестности точки $\widetilde{n}_{l}$ :

$$
t(n)=t_{l}, \quad \Delta(n)=\Delta_{l}+\gamma_{l}^{2} m
$$

где

$$
\begin{gathered}
\Delta_{l}=\pi l+O\left(l^{-1}\right), \quad \gamma_{l}^{2}=\frac{F a^{2}}{2 k_{\tilde{n}_{l}}} \\
t_{l}=I+\frac{1}{2 k_{\tilde{n}_{l}}}\left(\begin{array}{cc}
O\left(\gamma^{2}\right) & b_{\widetilde{n}_{l}}+O\left(\gamma^{2}\right) \\
a_{\widetilde{n}_{l}}+O\left(\gamma^{2}\right) & O\left(\gamma^{2}\right)
\end{array}\right) .
\end{gathered}
$$

Положим

$$
\hat{a}(n)=e^{i \Delta_{l} m \sigma_{3}} h(y), \quad y=\gamma_{l} m
$$


Функция $h$ удовлетворяет уравнению

$$
h(y+\gamma)=e^{i \gamma y \sigma_{3}} \hat{t} h(y),
$$

где

$$
\hat{t}_{l}=I+\frac{1}{2 k_{\tilde{n}_{l}}}\left(\begin{array}{cc}
O\left(\gamma^{2}\right) & b_{\widetilde{n}_{l}}\left(1+O\left(\gamma f^{-2}\right)\right) \\
a_{\widetilde{n}_{l}}\left(1+O\left(\gamma f^{-2}\right)\right) & O\left(\gamma^{2}\right)
\end{array}\right) .
$$

Здесь и далее индекс $l$ опушен.

Удобно выделить главный осциллируюший член, поэтому введем новую функцию

$$
g(y)=\exp \left\{-\frac{i}{2} y(y-\gamma) \sigma_{3}\right\} h(y) .
$$

Функция $g$ удовлетворяет уравнению

$$
g(y+\gamma)=\exp \left\{-\frac{i}{2} y(y-\gamma) \sigma_{3}\right\} \hat{t} \exp \left\{\frac{i}{2} y(y-\gamma) \sigma_{3}\right\} g(y) .
$$

Перепишем последнее уравнение в виде

$$
g(y+\gamma)=\left(I+\gamma^{2}\left(\begin{array}{cc}
0 & e^{-i y^{2} b} \\
e^{i y^{2} \bar{b}} & 0
\end{array}\right)+O\left(\gamma^{4}\right)\right) g(y),
$$

где введено обозначение

$$
b=\frac{b_{\widetilde{n}_{l}}}{F a^{2}}\left(1+O\left(\gamma f^{-2}\right)\right) .
$$

Решение уравнения (3.3) будем искать в виде формального ряда

$$
g(y)=\sum_{p \geqslant 0} \gamma^{p} g_{p}(y)
$$

Заменяя в (3.3) функцию $g(y+\gamma)$ рядом Тейлора и приравнивая коэффициенты при одинаковых степенях $\gamma$, получим уравнения на функции $g_{p}$, решая которые, найдем, что

$$
\gamma^{1}: g_{0}=\hat{g}_{0}
$$

- константа;

$$
\gamma^{2}: g_{1}=\hat{g}_{1}+\left(\begin{array}{cc}
0 & \bar{R}_{1} b \\
R_{1} \bar{b} & 0
\end{array}\right) \hat{g}_{0},
$$

где $\hat{g}_{1}-$ константа и

$$
\begin{gathered}
R_{1}=\int_{-\infty}^{y} e^{i t^{2}} d t \\
\gamma^{3}: g_{2}=\hat{g}_{2}-\frac{1}{2}\left(\begin{array}{cc}
0 & \bar{R}_{1}^{\prime} b \\
R_{1}^{\prime} \bar{b} & 0
\end{array}\right) \hat{g}_{0}+\left(\begin{array}{cc}
R_{2}|b|^{2} & 0 \\
0 & \bar{R}_{2}|b|^{2}
\end{array}\right) \hat{g}_{0}+\left(\begin{array}{cc}
0 & \bar{R}_{1} b \\
R_{1} \bar{b} & 0
\end{array}\right) \hat{g}_{1},
\end{gathered}
$$


где $\hat{g}_{2}-$ константа и

$$
R_{2}=\int_{-\infty}^{y} e^{-i t^{2}} d t \int_{-\infty}^{t} e^{i s^{2}} d s .
$$

Нетрудно заметить, что функции $R_{1}$ и $R_{2}$ удовлетворяют соотношению

$$
R_{2}(\infty)+\bar{R}_{2}(\infty)=\left|R_{1}(\infty)\right|^{2}
$$

причем

$$
R_{1}(\infty)=e^{i \frac{\pi}{4}} \sqrt{\pi}
$$

Таким образом,

$$
\begin{gathered}
g=\hat{g}_{0}+\gamma \hat{g}_{1}+\gamma^{2} \hat{g}_{2}+\left(\begin{array}{cc}
\gamma^{2} R_{2}|b|^{2} & \gamma \bar{R}_{1} b(1+O(\gamma)) \\
\gamma R_{1} \bar{b}(1+O(\gamma)) & \gamma^{2} \bar{R}_{2}|b|^{2}
\end{array}\right) \hat{g}_{0}+\cdots \\
\hat{a}(n)=e^{i \Delta_{l} m \sigma_{3}} \exp \left\{\frac{i}{2} y(y-\gamma) \sigma_{3}\right\} g(y)
\end{gathered}
$$

ТЕОРема 2. На интервалах $i_{l}$, заданных в (3.2), уравнение (3.1) имеет решение $\hat{a}(n)$, удовлетворяющее следующим условиям:

1) $\hat{a}(n)=e^{i \Delta_{l} m \sigma_{3}} \exp \left\{\frac{i}{2} \gamma^{2} m(m-1) \sigma_{3}\right\} g(\gamma m)$;

2) функиия $g(y)$ допускает асимптотическое разложение в виде (3.4).

Доказательство этой теоремы мы также опускаем и ссылаемся на книгу [12].

\section{4. ПЕРЕХОД ЧЕРЕЗ ТОЧКИ ПОВОРОТА}

Как видно из изложенного выше, мы построили асимптотические решения на интервалах $I_{l}$ и $i_{l}$, определенных формулами (2.8) и (3.2) соответственно. Нетрудно заметить, что интервал $i_{l}$ пересекается с двумя интервалами $I_{l}$ и $I_{l+1}$, поэтому построенные асимптотические решения можно сшить.

Сравним в области пересечения интервалов $i_{l}$ и $I_{l}$ адиабатические решения $(2.22)$ и решение (3.5). Асимптотика адиабатических решений на границе $i_{l}$ имеет вид

$$
\hat{a}_{l}^{(1)}(n)=e^{i \Theta(n)} e_{1}, \quad \hat{a}_{l}^{(2)}(n)=e^{-i \Theta(n)} e_{2}
$$

где функция $\Theta(n)$ определена в $(2.21)$. В области пересечения интервалов $i_{l}$ и $I_{l}$ имеем

$$
\begin{aligned}
\Theta(n) & =\Theta\left(n_{l}\right)+\Theta^{\prime}\left(n_{l}\right) m+\frac{1}{2} \Theta^{\prime \prime}\left(n_{l}\right) m^{2}+\cdots= \\
& =\Theta_{l}+\Delta_{l} m+\frac{1}{2} \gamma^{2} m(m-1)+O(\gamma), \\
\Theta_{l} & =\frac{2}{3 F} k_{n_{l}}^{3} .
\end{aligned}
$$

Введем адиабатические решения

$$
\omega^{(1)}(m)=e^{-i \Theta_{l}} \hat{a}_{l}^{(1)}(n), \quad \omega^{(2)}(m)=e^{i \Theta_{l}} \hat{a}_{l}^{(2)}(n) .
$$


На границе интервала $i_{l}$ эти решения имеют следуюшее асимптотическое поведение:

$$
\begin{aligned}
& \omega^{(1)}(m) \sim \exp \left\{i\left[\Delta_{l} m+\frac{1}{2} \gamma^{2} m(m-1)\right]\right\} e_{1}, \\
& \omega^{(2)}(m) \sim \exp \left\{-i\left[\Delta_{l} m+\frac{1}{2} \gamma^{2} m(m-1)\right]\right\} e_{2} .
\end{aligned}
$$

При положительных и отрицательных $m$ эти адиабатические решения строятся независимым образом. Обозначим их при $m<0$ через $u$ и при $m>0$ через $v$. Решение $u$ связано с $\hat{a}_{l}$, а решение $v-\mathrm{c} \hat{a}_{l+1}$. Внутренняя связь этих решений может быть фиксирована с помощью решения (3.5), определенного на интервале $i_{l}$. Для краткости обозначим это решение через $A$.

При $m \rightarrow-\infty$ имеем

$$
A \sim \exp \left\{i\left[\Delta_{l} m+\frac{1}{2} \gamma^{2} m(m-1)\right] \sigma_{3}\right\}\left(\hat{g}_{0}+\gamma \hat{g}_{1}+\gamma^{2} \hat{g}_{2}\right)
$$

При $m \rightarrow+\infty$

$$
\begin{aligned}
A \sim & \exp \left\{i\left[\Delta_{l} m+\frac{1}{2} \gamma^{2} m(m-1)\right] \sigma_{3}\right\}\left(\hat{g}_{0}+\gamma \hat{g}_{1}+\gamma^{2} \hat{g}_{2}\right)+ \\
& +\exp \left\{i\left[\Delta_{l} m+\frac{1}{2} \gamma^{2} m(m-1)\right] \sigma_{3}\right\} \times \\
& \times\left(\begin{array}{cc}
\gamma^{2} R_{2}(\infty)|b|^{2} & \gamma \bar{R}_{1}(\infty) b(1+O(\gamma)) \\
\gamma R_{1}(\infty) \bar{b}(1+O(\gamma)) & \gamma^{2} \bar{R}_{2}(\infty)|b|^{2}
\end{array}\right) \hat{g}_{0} .
\end{aligned}
$$

Введем координаты векторов $\hat{g}_{0}, \hat{g}_{1}, \hat{g}_{2}$ :

$$
\hat{g}_{0}=\left(\begin{array}{l}
\hat{g}_{01} \\
\hat{g}_{02}
\end{array}\right), \quad \hat{g}_{1}=\left(\begin{array}{l}
\hat{g}_{11} \\
\hat{g}_{12}
\end{array}\right), \quad \hat{g}_{2}=\left(\begin{array}{l}
\hat{g}_{21} \\
\hat{g}_{22}
\end{array}\right) .
$$

При $m \rightarrow-\infty$ решение $A$ может быть выражено в терминах $u^{(1)}$ и $u^{(2)}$ :

$$
\begin{aligned}
A=\{ & \left.\hat{g}_{01}+O\left(l^{-\frac{1}{2}}\right) \hat{g}_{11}+O\left(l^{-1}\right) \hat{g}_{21}\right\} u^{(1)}+ \\
& +\left\{\hat{g}_{02}+O\left(l^{-\frac{1}{2}}\right) \hat{g}_{12}+O\left(l^{-1}\right) \hat{g}_{22}\right\} u^{(2)}
\end{aligned}
$$

При $m \rightarrow+\infty$ решение $A$ может быть выражено в терминах $v^{(1)}$ и $v^{(2)}$ :

$$
\begin{aligned}
A=\{ & \hat{g}_{01}+O\left(l^{-\frac{1}{2}}\right) \hat{g}_{11}+O\left(l^{-1}\right) \hat{g}_{21}+\gamma^{2} R_{2}(\infty)|b|^{2} \hat{g}_{01}+ \\
& \left.+\left[\gamma \bar{R}_{1}(\infty) b\left(1+O\left(l^{-\frac{1}{2}}\right)\right)\right] \hat{g}_{02}\right\} v^{(1)}+\left\{\hat{g}_{02}+O\left(l^{-\frac{1}{2}}\right) \hat{g}_{12}+O\left(l^{-1}\right) \hat{g}_{22}+\right. \\
& \left.+\left[\gamma R_{1}(\infty) \bar{b}\left(1+O\left(l^{-\frac{1}{2}}\right)\right)\right] \hat{g}_{01}+\gamma^{2} \bar{R}_{2}(\infty)|b|^{2} \hat{g}_{02}\right\} v^{(2)}
\end{aligned}
$$


В результате получаем следующее равенство:

$$
\begin{aligned}
\left\{\hat{g}_{01}+O\left(l^{-\frac{1}{2}}\right) \hat{g}_{11}+O\left(l^{-1}\right) \hat{g}_{21}\right\} u^{(1)}+\left\{\hat{g}_{02}+O\left(l^{-\frac{1}{2}}\right) \hat{g}_{12}+O\left(l^{-1}\right) \hat{g}_{22}\right\} u^{(2)}= \\
=\left\{\hat{g}_{01}+O\left(l^{-\frac{1}{2}}\right) \hat{g}_{11}+O\left(l^{-1}\right) \hat{g}_{21}+\gamma^{2} R_{2}(\infty)|b|^{2} \hat{g}_{01}+\right. \\
\left.\quad+\left[\gamma \bar{R}_{1}(\infty) b\left(1+O\left(l^{-\frac{1}{2}}\right)\right)\right] \hat{g}_{02}\right\} v^{(1)}+\left\{\hat{g}_{02}+O\left(l^{-\frac{1}{2}}\right) \hat{g}_{12}+O\left(l^{-1}\right) \hat{g}_{22}+\right. \\
\left.\quad+\left[\gamma R_{1}(\infty) \bar{b}\left(1+O\left(l^{-\frac{1}{2}}\right)\right)\right] \hat{g}_{01}+\gamma^{2} \bar{R}_{2}(\infty)|b|^{2} \hat{g}_{02}\right\} v^{(2)} .
\end{aligned}
$$

Значение этого равенства состоит в том, что решение, которое асимптотически аппроксимируется линейной комбинацией функций $u^{(1)}$ и $u^{(2)}$, после продолжения через интервал $i_{l}$ переходит в линейную комбинацию функций $v^{(1)}$ и $v^{(2)}$.

Полагая $\hat{g}_{01}=1, \hat{g}_{11}=\hat{g}_{21}=\hat{g}_{02}=\hat{g}_{12}=\hat{g}_{22}=0$ и $\hat{g}_{02}=1, \hat{g}_{01}=\hat{g}_{11}=\hat{g}_{21}=\hat{g}_{12}=$ $\hat{g}_{22}=0$, получим

$$
\left(\begin{array}{l}
u^{(1)} \\
u^{(2)}
\end{array}\right)=\left(\begin{array}{cc}
1+\gamma^{2} R_{2}(\infty)|b|^{2} & \gamma R_{1}(\infty) \bar{b}(1+O(\gamma)) \\
\gamma \bar{R}_{1}(\infty) b(1+O(\gamma)) & 1+\gamma^{2} \bar{R}_{2}(\infty)|b|^{2}
\end{array}\right)\left(\begin{array}{l}
v^{(1)} \\
v^{(2)}
\end{array}\right)
$$

Отсюда следует, что

$$
\left(\begin{array}{l}
v^{(1)} \\
v^{(2)}
\end{array}\right)=M_{l}\left(\begin{array}{l}
u^{(1)} \\
u^{(2)}
\end{array}\right)
$$

где

$$
M_{l}=\left(\begin{array}{cc}
1+\gamma^{2} \bar{R}_{2}(\infty)|b|^{2} & -\gamma R_{1}(\infty) \bar{b}(1+O(\gamma)) \\
-\gamma \bar{R}_{1}(\infty) b(1+O(\gamma)) & 1+\gamma^{2} R_{2}(\infty)|b|^{2}
\end{array}\right) .
$$

Согласно (4.2) имеем

$$
\left(\begin{array}{c}
\hat{a}_{l}^{(1)} \\
\hat{a}_{l}^{(2)}
\end{array}\right)=e^{i \Theta_{l} \sigma_{3}}\left(\begin{array}{c}
u_{l}^{(1)} \\
u_{l}^{(2)}
\end{array}\right), \quad\left(\begin{array}{c}
\hat{a}_{l+1}^{(1)} \\
\hat{a}_{l+1}^{(2)}
\end{array}\right)=e^{i \Theta_{l} \sigma_{3}}\left(\begin{array}{c}
v_{l}^{(1)} \\
v_{l}^{(2)}
\end{array}\right) .
$$

Теперь с помощью (4.3) можно связать коэффициенты $\hat{a}_{l+1}$ и $\hat{a}_{l}$ :

$$
\left(\begin{array}{c}
\hat{a}_{l+1}^{(1)} \\
\hat{a}_{l+1}^{(2)}
\end{array}\right)=N_{l}\left(\begin{array}{c}
\hat{a}_{l}^{(1)} \\
\hat{a}_{l}^{(2)}
\end{array}\right),
$$

где

$$
N_{l}=e^{i \Theta_{l} \sigma_{3}} M_{l} e^{-i \Theta_{l} \sigma_{3}} .
$$

Используя формулу (4.1), найдем

$$
\Theta_{l}=\frac{2}{3 F}\left(E+F_{a n}\right)^{\frac{3}{2}},
$$

где $n_{l}$ - ближайшее целое число к решению $\widetilde{n}_{l}$ уравнения

$$
\sqrt{E+F a \widetilde{n}_{l}} a=\pi l .
$$

6 Теоретическая и математическая физика, т. 123, № 1, 2000 г. 
Раскладывая $\Theta_{l}$ в ряд Тейлора, имеем

$$
\Theta_{l}=\frac{2}{3 F}\left(E+F a \widetilde{n}_{l}\right)^{\frac{3}{2}}+\left(E+F a \widetilde{n}_{l}\right)^{\frac{1}{2}} a\left(n_{l}-\widetilde{n}_{l}\right)+O\left(l^{-1}\right) .
$$

Подставляя известное выражение для $\widetilde{n}_{l}$, получим окончательную формулу

$$
\Theta_{l}=-\frac{1}{3 F}\left(\frac{\pi l}{a}\right)^{3}+\pi l \frac{E}{F a}+\pi \ln _{l}+O\left(l^{-1}\right) .
$$

Последний неубывающий член $\pi l n_{l}$ в этой формуле может быть отброшен, т.к. он не дает вклада в матрицу $N_{l}$. Убывающий член $O\left(l^{-1}\right)$ может быть отнесен к матрице $M_{l}$. Таким образом, можно написать, что

$$
N_{l}=e^{i \Gamma_{l} \sigma_{3}} \widehat{M}_{l} e^{-i \Gamma_{l} \sigma_{3}}
$$

где введены следующие обозначения:

$$
\begin{aligned}
\Gamma_{l} & =-\frac{1}{3 F}\left(\frac{\pi l}{a}\right)^{3}+\pi l \frac{E}{F a}, \\
\widehat{M}_{l} & =\left(\begin{array}{cc}
1+\bar{q}(l) l^{-1} & i \bar{r}(l) l^{-\frac{1}{2}}\left(1+O\left(l^{-\frac{1}{2}} f^{-2}\right)\right) \\
-i r(l) l^{-\frac{1}{2}}\left(1+O\left(l^{-\frac{1}{2}} f^{-2}\right)\right) & 1+q(l) l^{-1}
\end{array}\right), \\
r(l) l^{-\frac{1}{2}} & =-i \gamma \bar{R}_{1}(\infty) \frac{\bar{a}_{\tilde{n}_{l}}}{F a^{2}}=-i e^{-i \frac{\pi}{4}} \frac{1}{(2 F a l)^{\frac{1}{2}}} \int_{0}^{a} q(t) e^{-i \frac{2 \pi l}{a} t} d t, \\
q(l) l^{-1} & =\gamma^{2} R_{2}(\infty) \frac{\left|\bar{a}_{\tilde{n}_{l}}\right|^{2}}{F^{2} a^{4}}=\frac{1}{2 \pi F a l}\left|\int_{0}^{a} q(t) e^{-i \frac{2 \pi l}{a} t} d t\right|^{2} \int_{-\infty}^{+\infty} e^{-i t^{2}} d t \int_{-\infty}^{t} e^{i s^{2}} d s .
\end{aligned}
$$

\section{5. МАТРИЦА ПЕРЕХОДА}

На интервале $I_{l}$ произвольное решение $\hat{a}$ уравнения (2.2) может быть представлено асимптотической формулой

$$
\hat{a}_{l}=\hat{s}_{l} \hat{a}_{l}^{(1)}+\hat{t}_{l} \hat{a}_{l}^{(2)}
$$

где $\hat{s}_{l}$ и $\hat{t}_{l}-$ константы.

Введем вектор

$$
\widehat{\mathbf{s}}_{l}=\left(\begin{array}{c}
\hat{s}_{l} \\
\hat{t}_{l}
\end{array}\right) .
$$

Учитывая соотношения (4.4) и (4.5), легко найти связь между $\widehat{\mathbf{s}}_{l+1}$ и $\widehat{\mathbf{s}}_{l}$ :

$$
\widehat{\mathbf{s}}_{l+1}=\widehat{W}_{l} \widehat{\mathbf{s}}_{l}
$$

где

$$
\widehat{W}_{l}=\left(N_{l}^{t}\right)^{-1}
$$


Теперь можно описать асимптотическое поведение решения уравнения (2.1) на интервале $I_{l}$. Введем вектор

$$
\mathbf{w}_{n}=\left(\begin{array}{c}
\theta(x, E+F a n) \\
\varphi(x, E+\text { Fan })
\end{array}\right) \text {. }
$$

Тогда на интервалах $[n a,(n+1) a] \subset I_{l}$ произвольное решение $\psi$ уравнения $(2.1)$ может быть записано в виде

$$
\psi=\left(e^{-\frac{1}{2} \ln k_{n} \sigma_{3}}\left(\hat{s}_{l} \hat{a}_{l}^{(1)}+\hat{t}_{l} \hat{a}_{l}^{(2)}\right), \mathbf{w}_{n}\right) .
$$

Здесь введено вешественное скалярное произведение

$$
(a, b)=a_{1} b_{1}+a_{2} b_{2}
$$

Используя асимптотические формулы (2.22), получим

$$
\begin{aligned}
\psi(x) \sim & \frac{\hat{s}_{l}}{\sqrt[4]{E+F a n}} \exp \left\{i \frac{2}{3 F}(E+F a n)^{\frac{3}{2}}\right\} \theta(x, E+F a n)+ \\
& +\hat{t}_{l} \sqrt[4]{E+F a n} \exp \left\{-i \frac{2}{3 F}(E+F a n)^{\frac{3}{2}}\right\} \varphi(x, E+F a n) \sim \\
\sim & \frac{s_{l}}{\sqrt[4]{E+F a n}} \exp \left\{i \frac{2}{3 F}(E+F a n)^{\frac{3}{2}}+i \sqrt{E+F a n}(x-n a)\right\}+ \\
& +\frac{t_{l}}{\sqrt[4]{E+F a n}} \exp \left\{-i \frac{2}{3 F}(E+F a n)^{\frac{3}{2}}-i \sqrt{E+F a n}(x-n a)\right\} \sim \\
\sim & \frac{s_{l}}{\sqrt[4]{F x}} \exp \left\{i \frac{2}{3 F}(E+F x)^{\frac{3}{2}}\right\}+\frac{t_{l}}{\sqrt[4]{F x}} \exp \left\{-i \frac{2}{3 F}(E+F x)^{\frac{3}{2}}\right\}
\end{aligned}
$$

где

$$
\left(\begin{array}{l}
s_{l} \\
t_{l}
\end{array}\right)=\left(\begin{array}{ll}
1 & 0 \\
0 & i
\end{array}\right)\left(\begin{array}{l}
\hat{s}_{l} \\
\hat{t}_{l}
\end{array}\right)
$$

Учитывая соотношение (5.1), найдем связь между векторами $\mathbf{s}_{l}$ и $\mathbf{s}_{l+1}$ :

$$
\mathbf{s}_{l+1}=W_{l} \mathbf{s}_{l},
$$

где матрица перехода $W_{l}$ дается формулой

$$
W_{l}=e^{-i \Gamma_{l} \sigma_{3}} S_{l} e^{i \Gamma_{l} \sigma_{3}}
$$

Вследствие того что функция $f$ может быть выбрана сколь угодно медленно убывающей, можно написать

$$
S_{l}=\left(\begin{array}{cc}
1+q(l) l^{-1} & r(l) l^{-\frac{1}{2}}\left(1+O\left(l^{-\frac{1}{2}}\right)\right) \\
\bar{r}(l) l^{-\frac{1}{2}}\left(1+O\left(l^{-\frac{1}{2}}\right)\right) & 1+\bar{q}(l) l^{-1}
\end{array}\right) .
$$

Теперь, учитывая теоремы 1 и 2, можно сформулировать следующую теорему. 
Теорема 6. Пусть $q(x)-$ периодический потенциал из класса $L_{1}[0, a]$, удовлетворяяющй условию

$$
\int_{0}^{a} q(t) d t=0
$$

Тогда уравнение

$$
-\psi^{\prime \prime}+q(x) \psi-F x \psi=E \psi
$$

имеет решение $\psi(x)$, допускающее следующее асимптотическое разложение на интервалах $I_{l}$ :

$$
\psi(x) \sim \frac{s_{l}}{\sqrt[4]{F x}} \exp \left\{i \frac{2}{3 F}(E+F x)^{\frac{3}{2}}\right\}+\frac{t_{l}}{\sqrt[4]{F x}} \exp \left\{-i \frac{2}{3 F}(E+F x)^{\frac{3}{2}}\right\}
$$

Асимптотические разложения на смежных интервалах $I_{l}$ и $I_{l+1}$ связаны преобразованием

$$
\mathbf{s}_{l+1}=W_{l} \mathbf{s}_{l}, \quad \mathbf{s}_{l}=\left(\begin{array}{c}
s_{l} \\
t_{l}
\end{array}\right)
$$

Учитывая асимптотику для $\psi$, можем заключить, что

$$
|\psi(x)|^{2} \sim \frac{\left|s_{l}\right|^{2}+\left|t_{l}\right|^{2}}{\sqrt{F x}}+\text { осциллирующие члены. }
$$

Рассмотрим интеграл

$$
\int_{n_{l-1} a}^{n_{l} a}|\psi(x)|^{2} d x
$$

Асимптотически (для больших $l$ ) мы можем вычислить этот интеграл, подставляя вместо $|\psi|^{2}$ главньй член из формулы (5.2) (вклад относительно маленьких интервалов $i_{l-1}$, $i_{l}$ и осциллируюших членов может быть отброшен):

$$
\int_{n_{l-1} a}^{n_{l} a}|\psi(x)|^{2} d x \sim \int_{n_{l-1} a}^{n_{l} a} \frac{\left|s_{l}\right|^{2}+\left|t_{l}\right|^{2}}{\sqrt{F x}} d x=\frac{2 \pi}{F a}\left\|\mathbf{s}_{l}\right\|^{2} .
$$

Значение этой формулы мы обсуждали во введении.

Благодарности. Автор выражает благодарность В. С. Буслаеву, который оказывал помощь при обсуждении задачи и чьи идеи, заимствованные из статьи [8], использовались в этой работе. 


\section{Список литературы}

[1] В. С. Буслаев. ТМФ. 1984. Т. 58. С. 233-243.

[2] В.С. Буслаев, Л. А. Дмитриева. ТМФ. 1987. Т. 73. С. 430-442.

[3] В. С. Буслаев, Л. А. Дмитриева. Алгебра и анализ. 1989. Т. 1. № 2. С. 1-29.

[4] J. Avron, J. Zak. J. Math. Phys. 1977. V. 18. № 5. P. 918-921.

[5] J. Avron, L. Gunter, J. Zak. Solid State Commun. 1975. V. 16. № 2. P. 189-191.

[6] A. Nenciu, G. Nenciu. J. Phys. A. 1981. V. 14. № 10. P. 2817-2827.

[7] P. Exner. J. Math. Phys. 1995. V. 36. P. 4561-4570.

[8] V.S.Buslaev. Am. Math. Soc. Transl. 1999. V. 189. P. 45-57.

[9] F. Delyon, B. Simon, B. Souillard. Ann. Inst. H. Poincaré. 1985. V. 42. № 3. P. 283-309.

[10] D. J. Gilbert, D. B. Pearson. J. Math. Anal. Appl. 1987. V. 128. P. 30-56.

[11] S. Khan, D. B. Pearson. Helv. Phys. Acta. 1992. V. 65. P. 505-527.

[12] В. Вазов. Асимптотические разложения решений обыкновенных дифференциальных уравнений. М.: Мир, 1968.

Поступила в редакцию 31.V.1999 г. 